\title{
miR-335-5p suppresses gastric cancer progression by targeting MAPK10
}

\author{
Yi Gao ${ }^{1,2 \dagger}$, Yanfeng Wang ${ }^{3 \dagger}$, Xiaofei Wang ${ }^{4 \dagger}$, Changan Zhao ${ }^{5}$, Fenghui Wang ${ }^{1,2}$, Juan Du ${ }^{1,2}$, Huahua Zhang ${ }^{1,2}$, \\ Haiyan Shi ${ }^{1,2}$, Yun Feng ${ }^{1,2}$, Dan Li $i^{1,2}$, Jing Yan ${ }^{1,2}$, Yan Yao ${ }^{1,2}$, Weihong Hu ${ }^{1,2}$, Ruxin Ding ${ }^{1,2}$, Mengjie Zhang ${ }^{1,2}$, \\ Lumin Wang ${ }^{4^{*}}$, Chen Huang ${ }^{4^{*}}$ and Jing Zhang ${ }^{1,2^{*}}$ (1)
}

\begin{abstract}
Background: Recent studies have established the roles of microRNAs (miRNAs) in cancer progression. The aberrant expression of miR-335-5p has been reported in many cancers, including gastric cancer (GC). In this study, the precise roles of miR-335-5p in GC as well as the molecular mechanisms underlying its effects, including the role of its target MAPK10, were evaluated.

Methods: Quantitative real-time PCR was used to evaluate miR-335-5p levels in GC cell lines and tissues. MTT and colony formation assays were used to detect cell proliferation, and Transwell and wound-healing assays were used to evaluate the invasion and migration of GC cells. The correlation between levels of miR-335-5p and the cell cyclerelated target gene mitogen-activated protein kinase 10 (MAPK10) in GC was analyzed. In addition, the candidate target was evaluated by a luciferase reporter assay, qRT-PCR, and western blotting.

Results: The levels of miR-335-5p were downregulated in GC tissues and cell lines. Furthermore, miR-335-5p inhibited the proliferation and migration of GC cells and induced apoptosis. Additionally, miR-335-5p arrested the cell cycle at the G1/S phase in GC cells in vitro. Levels of miR-335-5p and the cell cycle-related target gene MAPK10 in GC were correlated, and MAPK10 was directly targeted by miR-335-5p.
\end{abstract}

Conclusions: These data suggest that miR-335-5p is a tumor suppressor and acts via MAPK10 to inhibit GC progression.

Keywords: miR-335-5p, Gastric cancer, MAPK10, Proliferation

\section{Background}

Gastric cancer (GC) is still a significant public health problem worldwide [1, 2]. Over 1,000,000 new cases and an estimated 783,000 deaths were reported in 2018, making it the fifth most frequently diagnosed cancer and the

\footnotetext{
*Correspondence: wanglumin1@xjtu.edu.cn; hchen@xjtu.edu.cn; yadxzj@163.com

${ }^{+} Y_{i}$ Gao, Yanfeng Wang and Xiaofei Wang contributed equally to this work ${ }^{1}$ Department of Cell Biology and Genetics, Medical College of Yan'an University, Yan'an 716000, Shaanxi, China

${ }^{4}$ Department of Cell Biology and Genetics, School of Basic Medical Sciences, Xi'an Jiaotong University Health Science Center, Xi'an 710061, Shaanxi, China

Full list of author information is available at the end of the article
}

third leading cause of cancer deaths [3]. A wide range of factors, such as lifestyle, Helicobacter pylori infection, polyps, gastric ulcers, genetic factors, and gastric residual tissue, may be involved in gastric tumorigenesis [4]. Although there are many methods for the diagnosis and treatment of GC, 30\% of patients are diagnosed at an advanced stage [5]. Thus, useful biomarkers for early screening or detection are essential for improving survival rates [6].

Recent studies have established the important roles of microRNAs (miRNAs), which are small, non-proteincoding RNAs that regulate gene expression at the posttranscriptional level, in tumorigenesis [7-9]. Mature

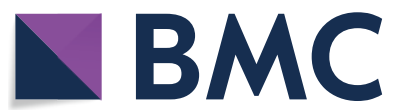

(c) The Author(s) 2021. This article is licensed under a Creative Commons Attribution 4.0 International License, which permits use, sharing, adaptation, distribution and reproduction in any medium or format, as long as you give appropriate credit to the original author(s) and the source, provide a link to the Creative Commons licence, and indicate if changes were made. The images or other third party material in this article are included in the article's Creative Commons licence, unless indicated otherwise in a credit line to the material. If material is not included in the article's Creative Commons licence and your intended use is not permitted by statutory regulation or exceeds the permitted use, you will need to obtain permission directly from the copyright holder. To view a copy of this licence, visit http://creativeco mmons.org/licenses/by/4.0/. The Creative Commons Public Domain Dedication waiver (http://creativecommons.org/publicdomain/ zero/1.0/) applies to the data made available in this article, unless otherwise stated in a credit line to the data. 
miRNAs of about 22 nucleotides contribute to gastric carcinogenesis by altering the expression of oncogenes and tumor suppressors [10]. For example, miR-181d [11], miR-99a [12], miR-105 [13], and others have suppressive effects on GC development, whereas other miRNAs, including miR-188-5p [14] and miR-221 [15], promote GC growth.

MiR-335-5p is abnormally expressed in many cancers. For instance, miR-335-5p is significantly downregulated and has a vital role in the metastasis of non-small cell lung cancer [16]. miR-335-5p is downregulated in breast cancer cells and is a promising biomarker for breast cancer treatment [17]. Additionally, miR-335-5p is downregulated in renal cell carcinoma and is a candidate therapeutic target [18]. The downregulation of miR-335 in GC has been reported [19]; however, its precise roles in GC cells are not fully understood.

MiRNAs function by binding to the $3^{\prime} \mathrm{UTR}$ of target mRNAs in a complementary base-pairing manner, thereby contributing to cell apoptosis, proliferation, and differentiation [20-23]. They are thought to regulate more than $50 \%$ of protein-coding genes. Based on a literature review and gene target prediction algorithms, including TargetScan, miRanda, and miRBase, we hypothesized that mitogen-activated protein kinase 10 (MAPK10) is a potential target of miR-335-5p. MAPK10 is a member of the Jun N-terminal kinase subgroup of mitogen-activated protein kinases. MAP kinases act as integration points for multiple biochemical signals and are involved in a wide variety of cellular processes, such as proliferation, differentiation, transcription regulation, and development $[24,25]$. Expression patterns of MAP kinases differ depending on the tumor type. Zhang et al. showed that MAPK10 is expressed at low levels in cervical cancer tissues and cells [26]. However, the percentage of MAPK10 protein-positive cells is significantly higher in ovarian serous, mucinous, and clear cell carcinomas than in normal tissues [27].

In this study, the effect of miR-335-5p on GC progression via MAPK10 was evaluated. In particular, we compared the expression levels of miR-335 in GC tissues and cells with those in matched normal tissues and MKN-28 and SGC-7901 cell lines. In addition, we used bioinformatics approaches, luciferase assays, qRT-PCR, and western blotting to evaluate its relationship with MAPK10 and further evaluated the functions of miR335 and MAPK10 in GC cell proliferation, metastasis, and apoptosis. Overall, our results demonstrated that miR-335 suppresses the progression of GCs by targeting MAPK10.

\section{Materials and methods}

\section{Cell lines and cell culture}

Human GC cell lines (AGS, BGC-823, MKN-45, MKN28, and SGC-7901), a normal gastric epithelial cell line (GES-1), and model cells (HEK-293) were provided by the Biomedical Experiment Center of Xi'an Jiaotong University (China). The use of these cell lines was approved by the Ethics Committee of Yan'an University College of Medicine (China). Human GC cells were cultured in DMEM (PAA Laboratories, Pasching, Australia) containing $10 \%$ fetal bovine serum and RPMI1 640 medium (PAA Laboratories) at $37^{\circ} \mathrm{C}$ in a $5 \% \mathrm{CO}_{2}$ incubator. The culture medium was changed once every 2-3 days. MKN-28 and SGC-7901 cells in the logarithmic growth phase were collected and subjected to the following experiments.

\section{Cell transfection}

GC cells in the logarithmic growth phase were digested and inoculated onto a 6-well culture plate. After cells reached $60-80 \%$ confluence, the miR-335-5p-mimics and miR-335-5p-inhibitor (GenePharma, Shanghai, China) were added to the corresponding wells for further culture for $24-48 \mathrm{~h}$.

\section{Quantitative real-time PCR}

RNA was extracted from GC cells using TRIzol. The cDNA was obtained by reverse-transcription using commercially available kits, according to the manufacturer's instructions. Quantitative real-time PCR was performed using the PrimeScript ${ }^{\mathrm{TM}}$ RT Reagent Kit (Takara Bio, Kusatsu, Japan) and an iQ5 Optical realtime PCR System (Bio-Rad, Hercules, CA, USA). The following primer sequences were used for amplification: RT miR-335-5p， 5'-GTCGTATCCAGTGCGTGTCGT GGAGTCGGCAATTGCACTGGATACGACacatttt-3'; RT U6 5'-CGCTTCACGAATTTGCGTGTCAT-3'; miR-335-5p, forward 5'-ATCCAGTGCGTGTCGTG-3' and reverse 5'-TGCTTCAAGAGCAATAACGA-3'; U6, forward 5'-GCTTCGGCAGCACATATACTAAAAT-3' and reverse 5'-CGCTTCACGAATTTGCGTGTCAT-3'; MAPK10 forward 5'-TTCTCAGGCACGGAATGG-3 and reverse 5'-TAAGTTGCCATAGTGAAGATCTGA G-3'; and glyceraldehyde-3-phosphate dehydrogenase $(G A P D H)$, forward 5'-TGAAGGTCGGAGTCAACG GATT $-3^{\prime}$ and reverse $5^{\prime}$-CCTGGAAGATGGTGATGG GATT-3'. The 20- $\mu \mathrm{L}$ PCR system consisted of $10-\mu \mathrm{L}$ of $2 \times$ RealStar Green Power Mixture, $1 \mu \mathrm{L}$ of forward primer $(10 \mu \mathrm{M}), 1 \mu \mathrm{L}$ of reverse-primer $(10 \mu \mathrm{M}), 2 \mu \mathrm{L}$ of cDNA, and $6 \mu \mathrm{L}$ of $\mathrm{ddH}_{2} \mathrm{O}$. The amplification conditions were as follows: $95{ }^{\circ} \mathrm{C}$ for $10 \mathrm{~min} ; 95{ }^{\circ} \mathrm{C}$ for 
$15 \mathrm{~s}, 60{ }^{\circ} \mathrm{C}$ for $1 \mathrm{~min}$, and $72{ }^{\circ} \mathrm{C}$ for $30 \mathrm{~s}$, for a total of 40 cycles. Relative expression levels of the target genes were calculated using the $2^{-\Delta \mathrm{Ct}}$ method. GAPDH was used as an internal reference.

\section{Western Blotting}

Cells were lysed with precooled radio-immunoprecipitation assay lysis buffer supplemented with protease inhibitor (Beyotime Institute of Biotechnology, Shanghai, China) for $30 \mathrm{~min}$ on ice. The supernatant was collected after centrifugation at $14,000 \mathrm{rpm}$ at $4{ }^{\circ} \mathrm{C}$ for $20 \mathrm{~min}$. The protein concentration was determined using a bicinchoninic acid protein concentration determination kit (RTP7102; Real-Times Biotechnology Co., Ltd., Beijing, China). The samples $(20 \mu \mathrm{g})$ were subjected to $10 \%$ sodium dodecyl sulfate-polyacrylamide gel electrophoresis and then transferred to polyvinylidene difluoride membranes. A GAPDH antibody was used as an internal reference. The membranes were washed with TBST and incubated with goat anti-mouse/rabbit antibody. Color development was performed using the chemiluminescence detection method, and images of protein bands were obtained for analyses. The luminescent signal was detected by a CCD camera, and recorded and quantified with Syngene GBox (Syngene, UK).The primary and secondary antibodies were as follows: anti-GAPDH monoclonal antibody (Cell Signaling Technology, Danvers, MA, USA; 5174S, diluted 1/1000), MAPK10 (Cell Signaling Technology; 2305S, diluted 1/1000), vimentin (Cell Signaling Technology; 5741S, diluted 1/1000), E-cadherin (ProteinTech, Rosemont, IL, USA; 20874-1-AP, diluted 1/1000), $\beta$-catenin (Cell Signaling Technology; 8480S, diluted 1/1000), CDK6 (Cell Signaling Technology; 13331S, diluted 1/1000), CDK4 (Cell Signaling Technology; 12790S, diluted 1/1000), CyclinD1 (Cell Signaling Technology; 55506S, diluted 1/1000), Bcl-2 (Cell Signaling Technology; 15071S, diluted 1/1000), Bax (ProteinTech; 50599-2-Ig, diluted 1/1000), goat anti-mouse immunoglobulin G (IgG) (ProteinTech; SA00001-1, diluted 1/3000), and goat anti-rabbit IgG (ProteinTech; SA00001-2, diluted 1/3000). Each western blotting was repeated at least three times.

\section{MTT assay}

Cell proliferation was assessed using the MTT Kit (Sigma, St Louis, MO, USA). Cells in the logarithmic growth phase were harvested and seeded on a 96-well plate. At 24, 48, and $72 \mathrm{~h}$ after seeding, $10 \mu \mathrm{L}$ of MTT was added to each well and the cells were incubated for $4 \mathrm{~h}$. Each well was supplemented with $150 \mu \mathrm{L}$ of DMSO, and the optical density (OD) was recorded at $490 \mathrm{~nm}$.

\section{Colony formation detection}

Transfected cells in the logarithmic growth phase were seeded onto a 6 -well plate. After 2 weeks of culture, the cells were fixed with $4 \%$ paraformaldehyde and stained with crystal violet. Images were obtained and cells were counted.

\section{Flow cytometry}

Transfected cells in the logarithmic growth phase were inoculated onto a 6-well plate and cultured for 1 day. Cells were fixed in $70 \%$ ethanol for $24 \mathrm{~h}$ and treated with propidium iodide and RNase provided with the kit. The cell cycle distribution was detected by flow cytometry.

\section{Dual luciferase reporter assay}

HEK-293 cells were divided into the miR-335-5p and pmirGLO empty vectors, miR-335-5p and pmirGLOMAPK10-WT (GenePharma), miR-335-5p, and pmirGLO- MAPK10-MuT (GenePharma) co-transfection groups, respectively. Untreated cells were used as controls. Wild-type and mutant MAPK10 were synthesized by GeneChem (Shanghai, China) as follows: wild-type MAPK10, up 5'-cATTTAACTTCTAGTT GCTCTTGCc- 3 ' and down 5'-tcgagGCAAGAGCAACT AGAAGTTAAATgagct-3'; and mutant MAPK10, up 5'-cATTTAACTTCTAGTTGATATCGCc-3' and down 5'-tcgagGCGATATCA ACTAGAAGTTAAATgagct-3'. Cells were inoculated onto 96-well plates and cultured for $24 \mathrm{~h}$. Luciferase activity was detected using a microplate reader. Renilla luciferase was used as an internal reference.

\section{Cell invasion assay}

Transwell chambers $(8-\mu \mathrm{m}$ pore size; Millipore, Billerica, MA, USA) were coated with Matrigel $(15 \mu \mathrm{g} /$ filter; BD Biosciences, Franklin Lakes, NJ, USA). Cells $\left(2.0 \times 10^{4}\right)$ in serum-free medium were added to the upper chamber, and the bottom wells were filled with complete medium. The cells were allowed to cross the Matrigel-coated membrane for $48 \mathrm{~h}$.

\section{Wound-healing assay}

A wound-healing assay was performed to examine metastasis. Briefly, after cells reached $90 \%$ confluence in 12 -well plates, a single scratch wound was generated with a $200-\mu \mathrm{L}$ disposable pipette tip. The extent of wound closure was measured after $48 \mathrm{~h}$.

\section{Statistical analysis}

Results are shown as means \pm SEM of at least three different experiments. The SPSS22.0 was used for statistical analyses. Bioinformatics analyses were performed using 
the ggstatsplot package in R. Experimental data were processed using GraphPad Prism7.0. Comparisons were conducted with the independent $t$-test. $P<0.05$ was considered statistically significant.

\section{Results}

miR-335-5p inhibits GC cell proliferation in vitro

To investigate the role and function of miR-335-5p in GC cells, we analyzed its expression in 22 pairs of GC tissues and matched adjacent non-cancerous tissue samples by qRT-PCR. miR-335-5p levels were significantly lower in GC samples than in non-cancerous tissue samples (Fig. 1a). These results were validated in five GC cell lines. miR-335-5p levels was lower in the BGC-823, SGC-7901, MKN-45, MKN-28, and AGS cell lines than in the GES-1 cell line (Fig. 1b). To clarify the function of miR-335-5p in GCs, MKN-28 and SGC-7901 cells were selected for further analyses. As determined by qRT-PCR, miR-335-5p mimics successfully elevated miR-335-5p expression in two cell lines; the effect of the inhibitor was moderate due to the low expression of endogenous miR-335-5p in MKN-28 and SGC-7901 cells (Fig. 1c). Thus, miR-335-5p may act as a tumor suppressor in GC.

miR-335-5p induces cell cycle arrest and apoptosis in GC

Gain- and loss-of-function analyses were conducted by transfecting MKN-28 and SGC-7901 cells with miR335-5p inhibitor-ctrl, inhibitor, miR-ctrl, and mimics. MTT and colony formation assays showed that the upregulation of miR-335-5p in MKN-28 and SGC-7901 cells inhibited cell growth and colony formation, while the inhibition of miR-335-5p exerted moderate adverse effects on GC cells, which may be explained by the low levels of miR-335-5p in MKN-28 and SGC-7901 cells (Fig. 2a, b). Consistent with these results, a flow cytometry analysis revealed that the upregulation of miR335-5p arrested cells in the G0/G1 phase and inhibited the transition to the $\mathrm{G} 2 / \mathrm{M}$ phase; similar effects were not observed in the miR-ctrl-transfected cells (Fig. 2c).
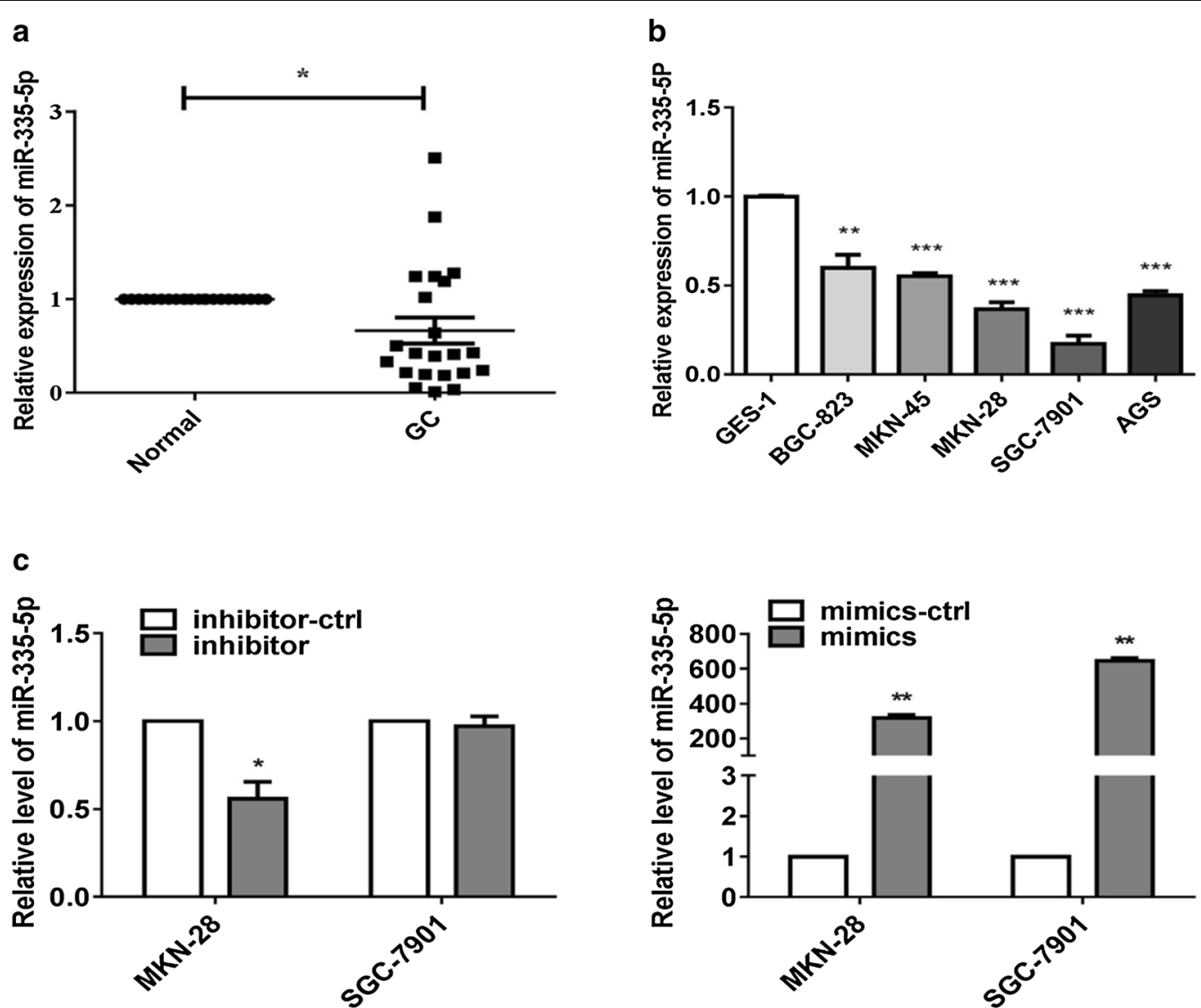

Fig. 1 Down-regulation of miR-335-5p in GC tissues and cells. a qRT-PCR analysis of miR-335-5p expression in 22 paired human gastric cancer and adjacent normal tissues. The expression of miR-335-5p was normalized to U6. $\mathbf{b}$ qRT-PCR analysis of miR-335-5p expression in normal gastric mucosal and gastric cancer cells and normalized against U6 RNA. c Expression levels of miR-335-5p were determined by qRT-PCR in GCs transfected with miR-335-5p mimics, inhibitor, or respective controls $\left({ }^{*} P<0.05\right.$, ${ }^{* *} P<0.01$, and $\left.{ }^{* * *} P<0.005\right)$ 

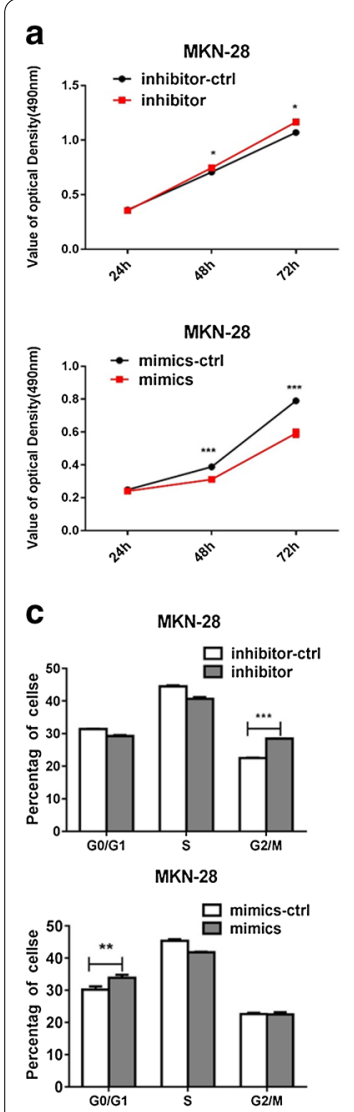
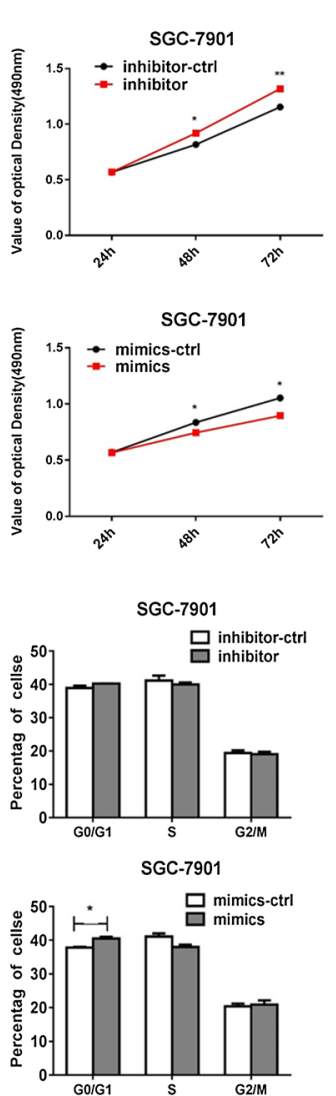
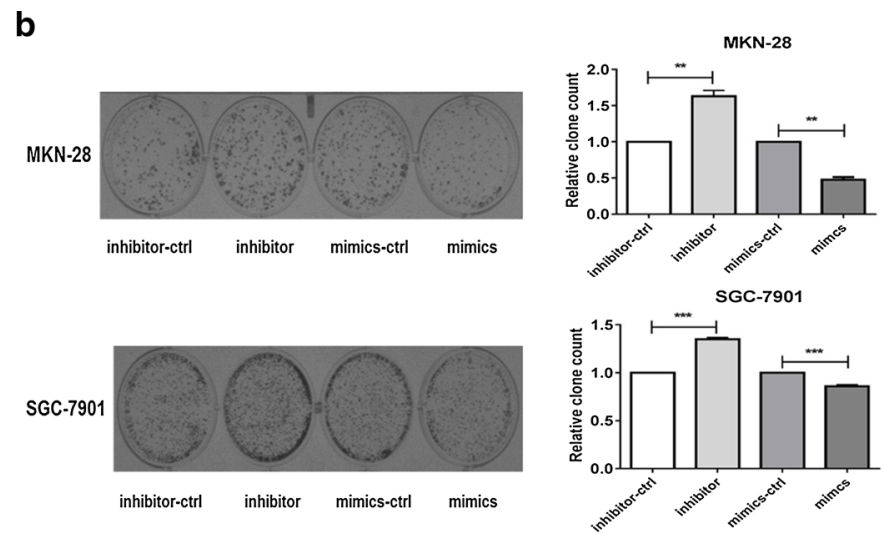

d
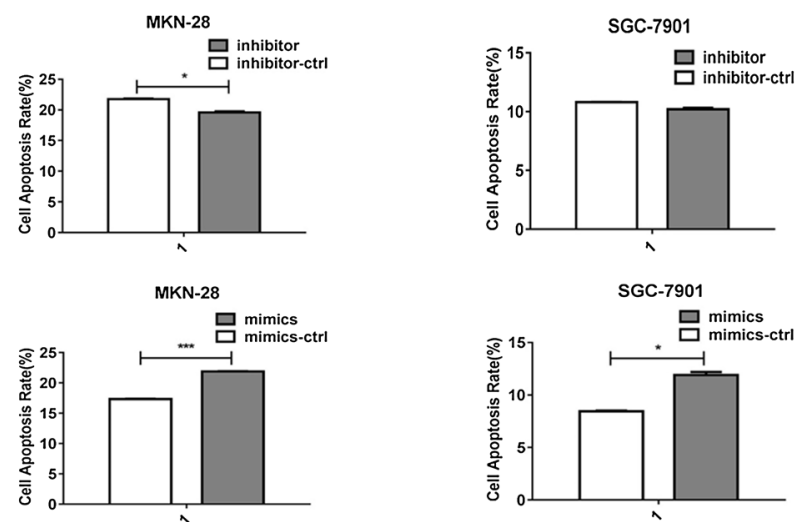

Fig. 2 miR-335-5p inhibited proliferation and promoted apoptosis in gastric cancer cells. a The effects miR-335-5p on gastric cancer cell proliferation were determined by an MTT assay after the transfection of MKN-28/SGC-7901 cells with an miR-335-5p mimic or miR-335-5p inhibitor at 24, 48, and 72 h. b The growth of MKN-28/SGC-7901 cells was detected by colony formation after transfection with the miR-335-5p mimic or inhibitor. c Cell cycle progression was evaluated in MKN-28/SGC-7901 cells transfected with miR-335-5p inhibitor-ctrl, inhibitor miR-335-5p ctrl, and mimics. After $48 \mathrm{~h}$, the cell cycle distribution was analyzed by flow cytometry. The histogram indicates the percentages of cells in G0/G1, S, and G2/M phases. $\mathbf{d}$ Apoptosis was detected by annexin-V/propidium iodide combined labeling flow cytometry in MKN-28/SGC-7901 cells $48 \mathrm{~h}$ after transfection with miR-335-5p inhibitor-ctrl, inhibitor, miR-335-5p ctrl, and mimics. Apoptosis was evaluated as the percentage of apoptotic cells ( ${ }^{*} P<0.05$, ${ }^{* *} P<0.01$, and $\left.{ }^{* * *} P<0.005\right)$

Furthermore, flow cytometry confirmed that the upregulation of miR-335-5p induces apoptosis in GC cells. However, the miR-335-5p inhibitor resulted in a slight but non-significant difference in apoptosis compared to that in cells transfected with the negative control, which may be explained by the low expression level and low inhibitory efficiency in MKN-28 and SGC-7901 cells (Fig. 2d). The inhibition of MiR-335-5p promoted proliferation and inhibited apoptosis in GC cells, while the inverse results were obtained in the miR-335-5p mimic group.

\section{Inhibition of miR-335-5p induces the migration and invasion of gastric cancer cells}

To further confirm that miR-335-5p acts as a tumor suppressor, its effects on the invasion of MKN-28 and SGC7901 cells were evaluated by a Transwell invasion assay and wound-healing assay. In the wound-healing assay, migration was slower in the miR-335-5p-transfected cells than in un-transfected cells. Over time, the difference in the metastasis rate between the two groups increased (Fig. 3a). In the Transwell invasion assay, the transfection of cells with miR-335-5p mimics significantly impaired invasion compared to that in the miR-335-5p-ctrl group in MKN-28 and SGC-7901 cells. In contrast, the knockdown of miR-335-5p enhanced GC cell invasion. When transfected with the mir-335-5p inhibitor, the MKN-28 and SGC-7901 cell invasion rates increased significantly (Fig. 3b). These results support the hypothesis that miR335-5p contributes to the suppression of invasion and metastasis. To investigate the mechanisms underlying the roles of miR-335-5p in apoptosis and cell cycle progression, we measured the expression levels of apoptosis- and cell cycle-related proteins in GC cells. The transfection of MKN-28/SGC-7901 cells with MiR-335 

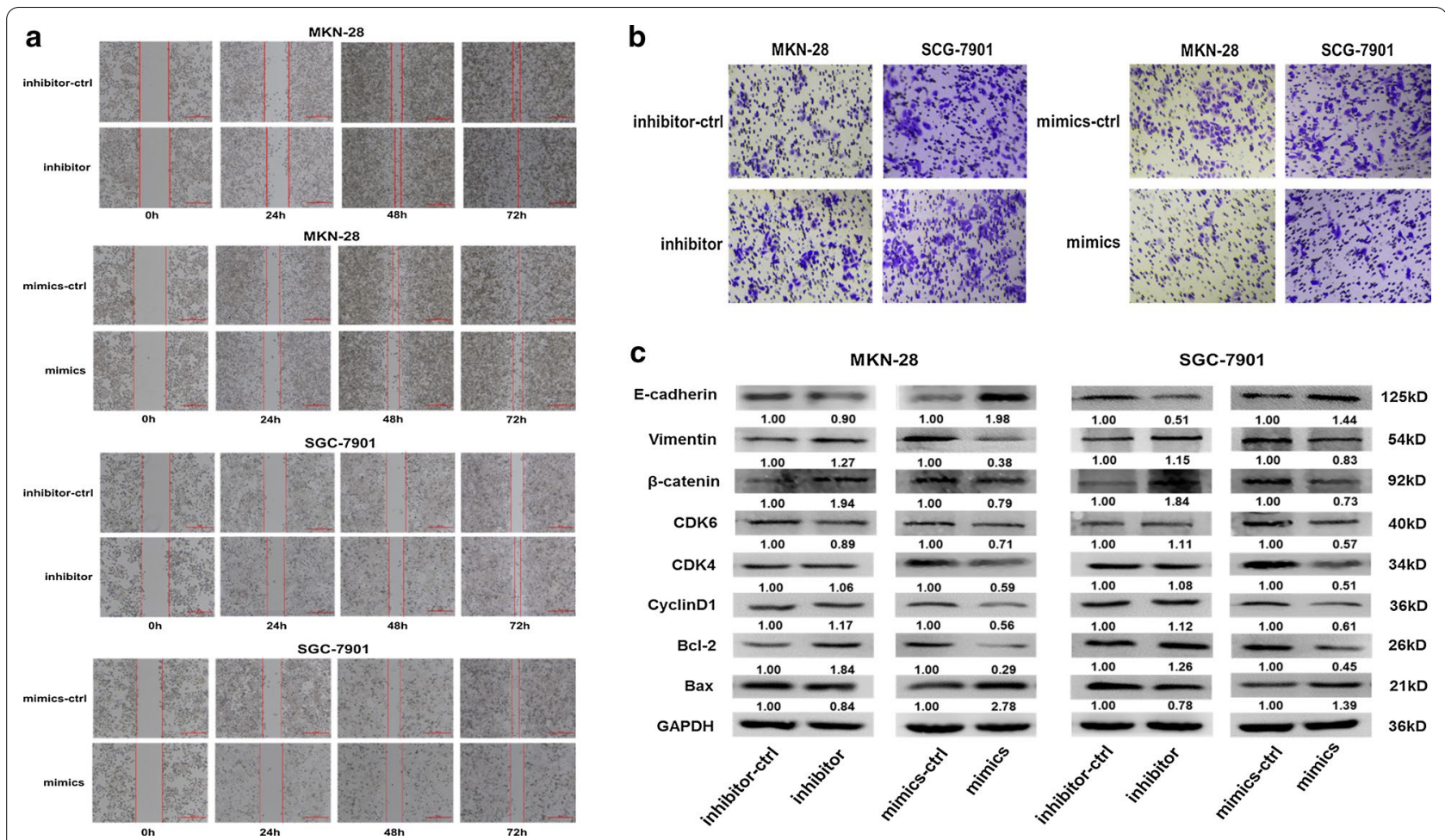

Fig. 3 miR-335-5p inhibited the migration and invasion of MKN-28 and SGC-7901 cells. a Scratch wound-healing assays of MKN-28 and SGC-7901 cells after treatment with miR-335-5p inhibitor-ctrl, inhibitor, miR-ctrl, or miR-335-5p mimics. b Transwell analysis of MKN-28 and SGC-7901 cells after transfection with miR-335-5p mimics, inhibitor, or their respective controls. c Western blot analysis of CDK6, CDK4, CyclinD1, BCL-2, BAX, E-cadherin, Vimentin, and $\beta$-catenin expression in MKN-28 and SGC-7901 cells transfected with miR-335-5p, inhibitor, or their respective controls

downregulated CDK6, CDK4, CyclinD1, and BCL-2 and upregulated the expression of BAX. The overexpression of miR-335-5p reduced the expression levels of vimentin and $\beta$-catenin, and significantly increased E-cadherin levels in MKN-28 and SGC-7901 cells. Our results showed that the silencing of miR-355-5p significantly increased the relative expression levels of vimentin and $\beta$-catenin and decreased E-cadherin expression, comparable with the effects of miR-355-5p overexpression in MKN-28 and SGC-7901 cells (Fig. 3c). These results suggest that mir-335-5p is involved in the progression, migration, and invasion of GCs.

\section{MAPK10 is a direct functional target of miR-335-5p in GC cells}

miRNA target prediction algorithms were used to search for potential miR-335-5p target genes. Levels of MAPK10 and mir-335-5p expression in GC based on TCGA data showed a negative correlation $(P<0.001$; Fig. $4 a)$. MAPK10 had a potential miR-335-5p-binding site in the $3^{\prime}$-UTR and therefore was selected as a candidate target. To determine whether MAPK10 was directly targeted by miR-335-5p, we subcloned 3'-UTR MAPK10 fragments including wild-type (MAPK10-WT) and mutant
(MAPK10-MUT) miR-335-5p-binding sites into the pmiRGLO dual-luciferase reporter vector (Fig. 4b). PremiR-335 and MAPK10-WT- or MUT-3'-UTR vectors were co-transfected into HEK293 cells. The relative luciferase activity of the MAPK10-WT pmirGLO-3-UTR vector was significantly reduced in miR-335-overexpressing HEK293 cells. As expected, miR-335-5p failed to inhibit the luciferase activity of the MAPK10-MUT pmirGLO$3^{\prime}$-UTR vector, indicating that miR-335-5p binds directly to the $3^{\prime}$-UTR of MAPK10 (Fig. 4c). qRT-PCR was used to verify the relationship between miR-335-5p and MAPK10. The mRNA levels of MAPK10 decreased significantly by miR-335 mimics and increased by miR-335 inhibitors in MKN-28 and SGC-7901 cells (Fig. 4d). The protein levels of MAPK10 decreased significantly by miR-335 mimics and increased by miR-335 inhibitors in MKN-28 and SGC-7901 cells (Fig. 4e). These findings demonstrated that miR-335-5p could directly target MAPK10 and suppress its expression in GC cells.

\section{Bioinformatics analysis of MAPK10 in gastric cancer}

The TCGA database was used to elucidate the effect of MAPK10 in GC tissues by a bioinformatics approach. The expression of MAPK10 was higher in GC tissues 

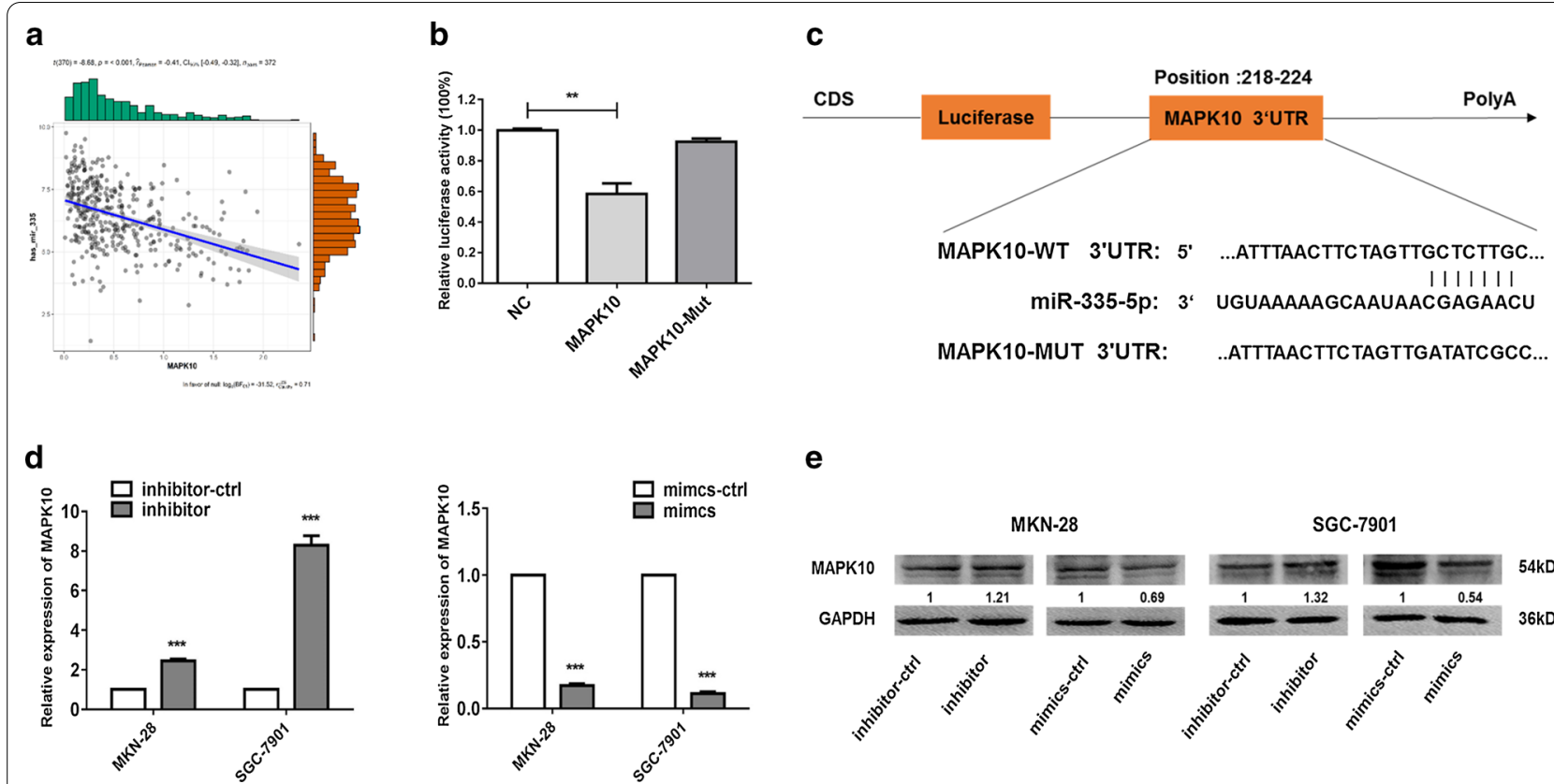

e

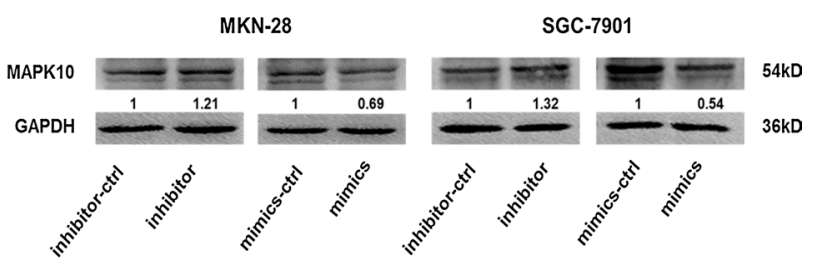

Fig. 4 MAPK10 is a direct target of miR-335-5p in gastric cancer cell lines. a Correlation between MAPK10 expression and miR-335-5p expression in gastric cancer based on TCGA data. b A luciferase assay was performed using HEK293 cells in which miR-335 was co-transfected with the pGLO-MAPK10 wild-type or pGLO-MAPK10 mutant vector. c miR-335-5p is highly conserved across species and has binding sites within the 3'-UTR of human MAPK10. $\mathbf{d}$ mRNA expression levels of MAPK10 were measured by qRT-PCR after transfection with miR-335-5p mimics, miR-335-5p inhibitor, or their negative controls in MKN-28 and SGC-7901 cells. e Protein expression levels of MAPK10 were measured by western blotting after transfection with miR-335-5p mimics, miR-335-5p inhibitor, or their respectively negative controls in MKN-28 and SGC-7901 cells $\left({ }^{*} P<0.05\right.$,

${ }^{*} P<0.01$, and $\left.{ }^{* *} P<0.005\right)$

than in healthy counterparts, and its expression was associated with the histologic and pathologic stages of GC (Fig. 5a-c). The expression of MAPK10 was associated with the DFI (disease-free interval, $P=0.033$ ), PFI (progression-free interval, $P=0.013$ ), DSS (disease-specific survival, $P=0.0068$ ), and OS (overall survival, $P=0.017$ ) in GC (Fig. $5 \mathrm{~d}-\mathrm{g}$ ), suggesting that MAPK10 plays a key role as an oncogene in $\mathrm{GC}$.

\section{Knockdown of MAPK 10 reduces $\mathrm{GC}$ progression}

We knocked down MAPK10 expression by RNA interference [small interfering RNA (siRNA)] to confirm that MAPK10 mediates the antitumor effects of miR335-5p. MAPK10 expression levels were higher in GC cells than in GES-1 cells (Fig. 6a) and were highest in MKN-28 and SGC-7901 cells. Western blotting indicated that the MAPK10 was obviously up-regulated in GC tissues than in their counterparts at the protein level (Fig. 6b). MAPK10 was successfully knocked down by siRNA, as verified by analyses of both at the mRNA levels (Fig. 6c). Similar to miR-335-5p-overexpressing cells, the downregulation of MAPK10 significantly inhibited proliferation and slightly inhibited colony formation in MKN-28 and SGC-7901 cells (Fig. 6d, e). Moreover, the influence of MAPK10 siRNA on the cell cycle was similar to the effect of miR-335-5p upregulation (Fig. 6f). Consistent with the effect of miR-335-5p on GC cell apoptosis, MAPK10 knockdown induced apoptosis in MKN28/ SGC-7901 cells (Fig. 6g), suggesting that MAPK10 is involved in the progression of GC.

\section{Knockdown of MAPK10 reduces the migration and invasion of GC cells}

We silenced MAPK10 expression by RNA interference (RNAi) to evaluate whether it contributes to the effects of miR-335-5p on invasion and metastasis using MKN28 and SGC-7901 cells. Based on a wound-healing assay, the group with low MAPK10 expression showed reduced rates of migration (Fig. 7a). Transwell assays demonstrated that MAPK10 silencing inhibited the invasion and migration ability of GC cells (Fig. 7b). Based on a western blot analysis, silencing MAPK10 significantly increased the relative expression levels of E-cadherin and decreased vimentin and $\beta$-catenin expression. These results were consistent with the effects of miR-355-5p overexpression in MKN-28 and SGC-7901 cells (Fig. 7c), suggesting that MAPK10 functions as an oncogene in GC. We concluded that miR-335-5p suppresses GC progression by targeting MAPK10 (Fig. 7d). 


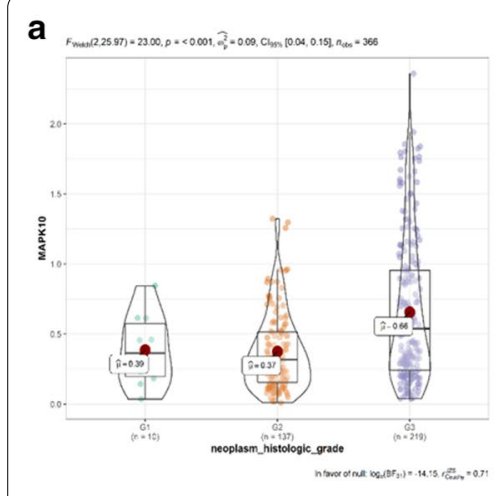

d ${ }_{D F I}$

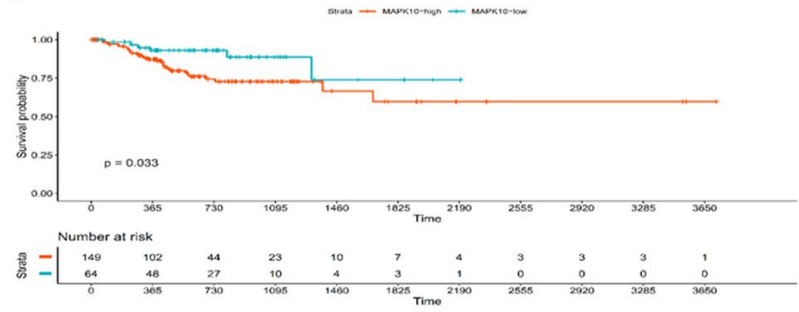

f os

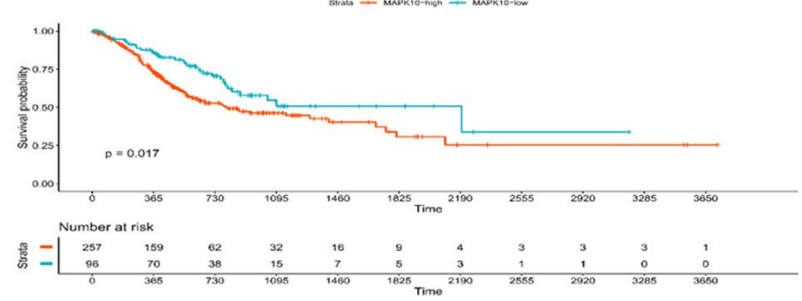

b
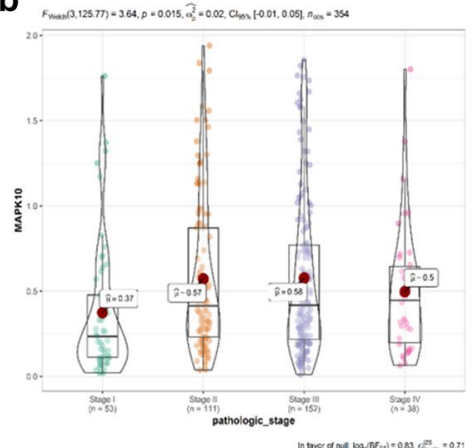

e oss
C

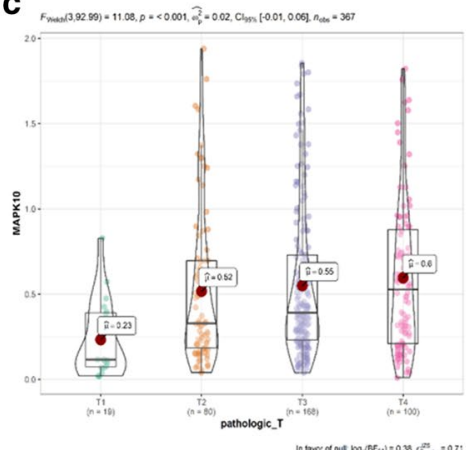

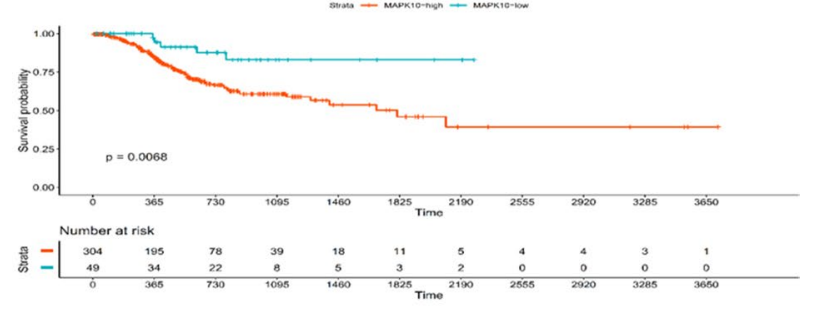

g PFI

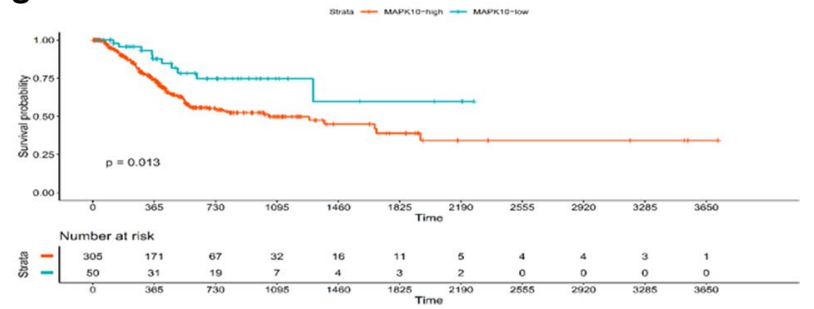

Fig. 5 a-c The expression of MAPK10 is associated with the pathologic and histologic grade of GC. $\mathbf{d}-\mathbf{g}$ Bioinformatics analyses were used to elucidate the effect of MAPK10 in GC tissues. The expression of MAPK10 was related to the DFI (disease-free interval event), PFI (progression-free interval event), DSS (disease-specific survival event), and OS (overall survival)

\section{Discussion}

The occurrence of GC is a complex process involving multiple factors, including genetic and epigenetic events. Many miRNAs have been demonstrated to contribute to GC tumorigenesis and development, and these serve as a valid diagnostic and therapeutic targets [28]. In addition, some specific microRNAs able to regulate the expression of genes in GC cells at the post-transcriptional level have been identified as diagnostic biomarkers for GC [29-31]. Therefore, studies of potential miRNAs associated with the development of GC may provide opportunities for improvements in diagnosis, treatment, and prognosis.

In this study, the roles of mir-335-5p in GC were evaluated. Recent studies have shown that miR-335-5p acts as a tumor suppressor and inducer in several cancer types [32-35]. For example, miR-335-5p is downregulated in thyroid cancer cells and inhibits proliferation [36]. miR335-5p functions via lactate dehydrogenase $B$ to exert tumor inhibitory effects in colorectal cancer [37]. In the present study, based on expression analyses of miR$335-5 p$ in tissues, we found that miR-335-5p acts as a tumor suppressor in GC, and the overexpression of miR335-5p inhibits proliferation, invasion, and metastasis and induces apoptosis in vitro. Additionally, miR-335-5p could induce cell cycle arrest at the G1 phase and downregulated the $\mathrm{G} 0$ and $\mathrm{G} 1$ phase cycle-associated proteins Cyclin D1, CDK 6, and CDK4. The abnormal activation of CDK and its modulators has been reported in many tumors $[38,39]$. Furthermore, miRNAs participate in the regulation of the cell cycle [40, 41]. For example, the miR$15 \mathrm{a} / 16$ family regulates $\mathrm{G0} / \mathrm{G} 1$ cell cycle progression by targeting cyclin D1 (CCND1) [42] addition, miR-16 regulates various mRNA targets, including CDK6, CDC27, and G1-related cyclins, which jointly control cell cycle progression [43]. These studies strongly support our observation that miR-335-5p plays a key role in cell proliferation in GC. 


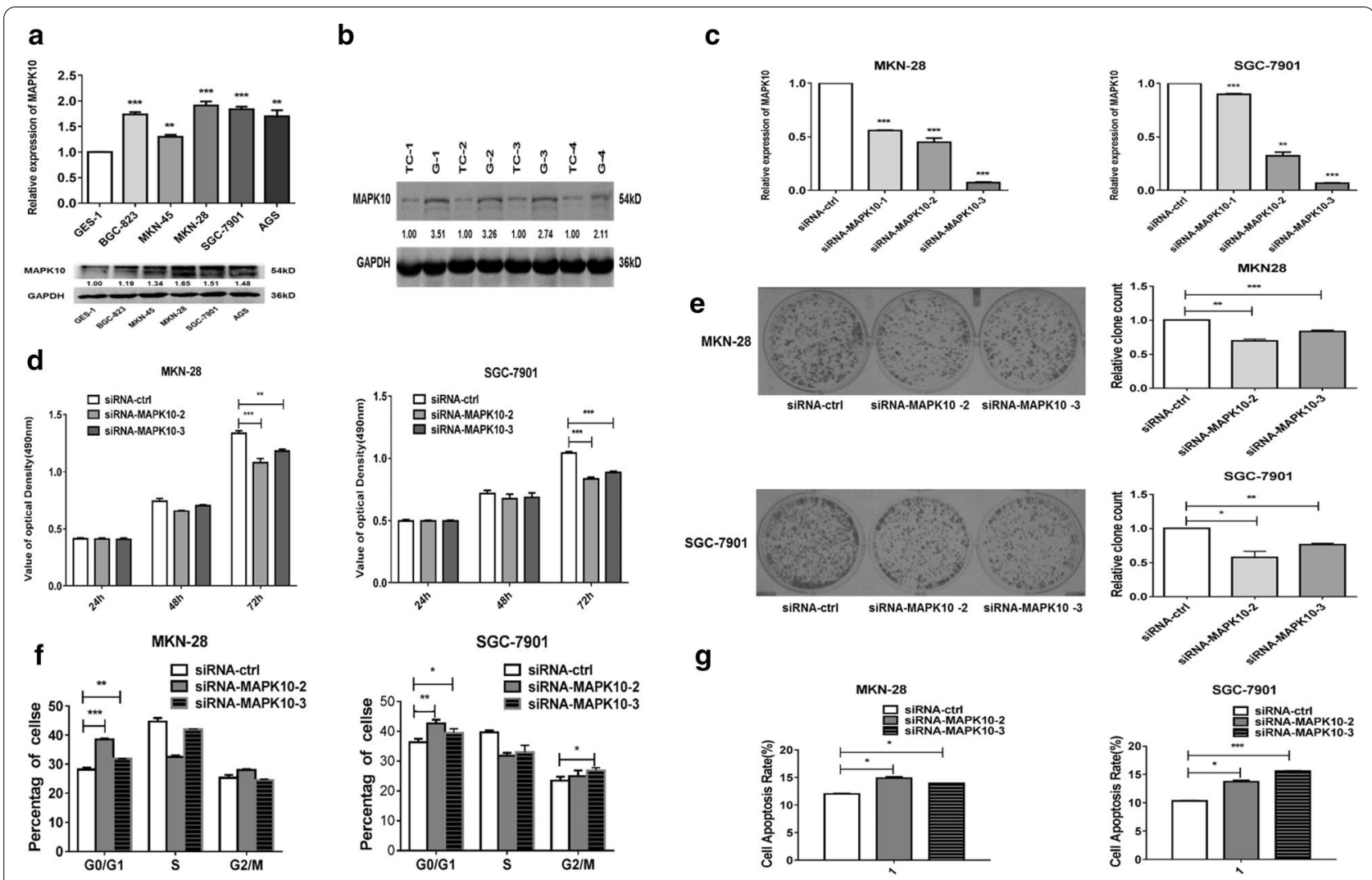

Fig. 6 Inhibition of MAPK10 suppressed GC progression. a mRNA and protein expression levels of MAPK10 in various GC and GES-1 cells. b MAPK10 protein expression in GC tissues vs counterparts'tissues was confirmed by using western blotting. $\mathbf{c}$ Expression levels of MAPK10 were measured by qRT-PCR in MKN-28 and SGC-7901 cells transfected with siMAPK10. d An MTT assay was performed to determine the growth of gastric cancer cells treated with siMAPK10 or a negative control (si-ctrl). e A colony formation assay was performed several days after the transfection of gastric cancer cells with siMAPK10 or a negative control (si-ctrl). f The cell cycle distribution was determined in gastric cancer cells $48 \mathrm{~h}$ after transfection with siMAPK10 by propidium iodide staining and flow cytometry. The histogram indicates the percentages of cells in G0/G1, S, and G2/M cell cycle phases. $g$ Apoptosis was determined in gastric cancer cells at $48 \mathrm{~h}$ after transfection with siMAPK10 $\left({ }^{*} P<0.05,{ }^{* *} P<0.01\right.$, and $\left.{ }^{* * *} P<0.005\right)$

Migration and invasion are closely related to the occurrence and development of tumors. Moreover, many migration- and invasion-related proteins, including E-cadherin, vimentin, and $\beta$-catenin, are involved. For instance, p0071 interacts with E-cadherin in the cytoplasm and promotes invasion and metastasis in non-small cell lung cancer [44]. Furthermore, ubiquitin specific peptidase 20 (USP20) regulates the deubiquitination of $\beta$-catenin to control the invasion and migration of cancer cells [45]. Our results showed that the overexpression of mir-335-5p decreases the expression of vimentin and $\beta$-catenin and increases E-cadherin in MKN-28 and SGC-7901 cells. Our data suggest that mir-335-5p is involved in the migration and invasion of GCs.

In addition, our results showed that the upregulation of miR-335-5p inhibits the expression of MAPK10 in MKN-28/SGC-7901 cancer cells at the RNA and protein levels. Using bioinformatic analyses and a dual-luciferase reporter assay, we demonstrated that miR-335-5p directly targets MAPK10 by binding to its $3^{\prime}$-UTR and inhibiting translation. To further clarify the tumor suppressive effect of miR-335-5p via MAPK10, siRNA was used. MAPK10 silencing inhibited cell proliferation and migration and induced cell apoptosis, similar to the observed effects of miR-335-5p overexpression in GC cells in vitro. Accordingly, the expression levels of related proteins, including CDK6, CDK4, CyclinD1, BCL-2, BAX, E-cadherin, vimentin, and $\beta$-catenin were also altered by siMAPK10. MAPK10 is a member of the Jun N-terminal kinase subgroup of the mitogen-activated protein kinases, which are implicated in important physiological processes [46]. MAPK10 regulates the occurrence and development of several types of cancer. The downregulation of MAPK10 contributes to the suppression of ovarian cancer [47]. miR-27a-3p promotes the growth and invasion of NPC cells by targeting Mapk10 [48]. These 

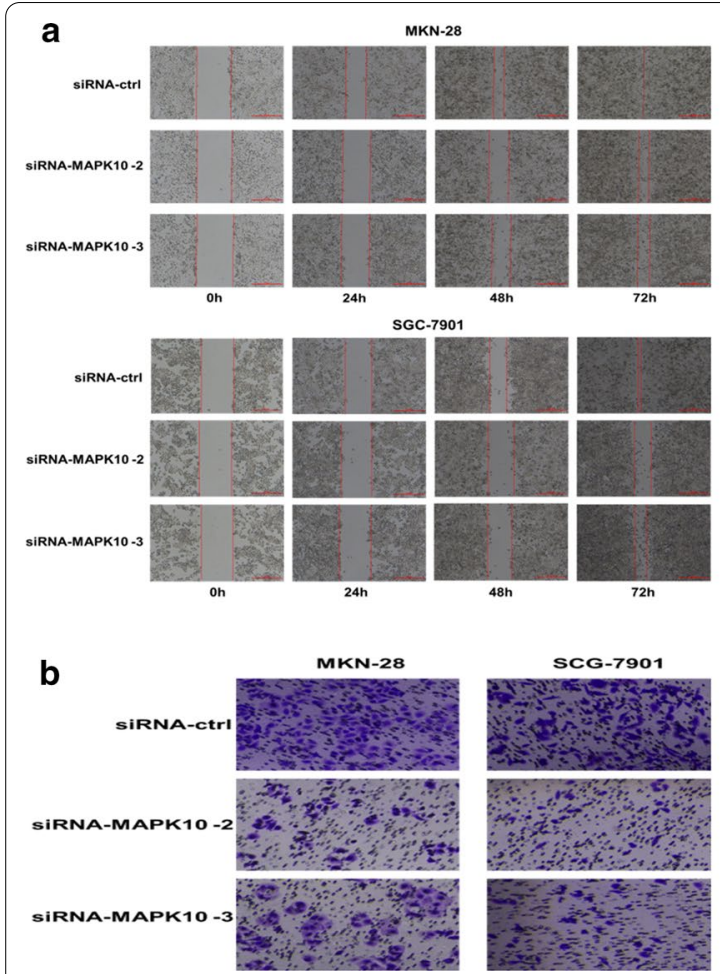

Fig. 7 miR-335-5p inhibited cell invasion via MAPK10 knockdown. a Wound-healing assays of MKN-28 and SGC-7901 cells after treatment with si-ctrl and si-MAPK10. Representative images were captured at 0 h, 24 h, 48 h, and $72 \mathrm{~h}$ after transfection with si-ctrl and si-MAPK10. b The invasion viability of MKN-28 and SGC-7901 cells transfected with siMAPK10 was determined by a Transwell invasion assay. c The expression levels of MAPK10 were measured by western blotting in MKN-28/SGC-7901 cells transfected with siMAPK10. Protein expression levels of CDK6, CDK4, CyclinD1, BCL-2, BAX, E-cadherin, Vimentin, and $\beta$-catenin in gastric cancer cells transfected with siMAPK10 or si-ctrl were analyzed by western blotting. $\mathbf{d}$ Proposed model for the suppressive effect of miR-335-5p on gastric cancer progression via MAPK10 knockdown

results robustly suggested that the downregulation of MAPK10 induced by miR-335-5p could inhibit GC progression. Our findings highlight that mir-335-5p or MAPK10 may be considered as potential targets for GC therapy in the near future.

\section{Conclusion}

We obtained the following new findings. (1) miR335-5p functions as a tumor suppressor in GC. (2) miR-335-5p leads to the inhibition of proliferation and metastasis and the promotion of apoptosis in GC cells. (3) MAPK10 is a downstream target gene of miR335-5p. (4) MAPK10 has a vital role as an oncogene in GC.

\section{Abbreviations}

miRNAs: MicroRNAs; GC: Gastric cancer; MAPK10: Mitogen-activated protein kinase 10; GAPDH: Glyceraldehyde-3-phosphate dehydrogenase; TBST: Tris buffer solution with tween; siRNA: Small interfering RNA.

\section{Acknowledgements}

We thank all the members of the Biomedical Experimental Center of Xi'an Jiaotong University for the discussion and support and for the critical reading of the manuscript.

\section{Authors' contributions}

YG and YW and LW: prepared the manuscript. XW, CZ, FW, JD, HZ, HS, YF, DL, $J Y, Y Y, W H, M Z, R D$ : collected the data and performed the statistical analysis. JZ and $\mathrm{CH}$ : conceived and supervised the study. All authors read and approved the final manuscript.

\section{Funding}

This work was supported by the National Natural Science Foundation of China (Grant No. 81660492, 81860444 and 81760510), Shaanxi Province Key Science and Technology innovation team (2020TD-039), Shaanxi Innovative Talents Promotion Plan-Science and Technology New Star Project (Grant No. 2017KJXX-20), Scientific Research Plan Projects of Yan'an University (Grant No. YDBK2018-49).

\section{Availability of data and materials}

The datasets supporting the conclusions of this study are included in this article. Any requests for data or materials can be sent to the corresponding author.

\section{Ethics approval and consent to participate} Not applicable. 


\section{Consent for publication \\ Not applicable.}

\section{Competing interests}

The authors declare that the research was conducted in the absence of any commercial or financial relationships that could be construed as a potential conflict of interest. The authors declare that they have non-financial conflict of interest.

\section{Author details \\ 1 Department of Cell Biology and Genetics, Medical College of Yan'an University, Yan'an 716000, Shaanxi, China. ${ }^{2}$ Yan'an Key Laboratory of Chronic Disease Prevention and Research, Yan'an 716000, Shaanxi, China. ${ }^{3}$ Depart- ment of Medical Genetic and Cell Biology, Ningxia Medical University, Yinchuan 750004, China. ${ }^{4}$ Department of Cell Biology and Genetics, School of Basic Medical Sciences, Xi'an Jiaotong University Health Science Center, Xi'an 710061, Shaanxi, China. ${ }^{5}$ Department of Pathology, School of Basic Medical Sciences, Xi'an Jiaotong University, Xi'an, China.}

Received: 10 August 2020 Accepted: 27 November 2020

Published online: 22 January 2021

\section{References}

1. Crew KD, Neugut Al. Epidemiology of gastric cancer. World J Gastroenterol. 2006;12(3):354-62.

2. Lau HCH, Kranenburg O, Xiao H, Yu J. Organoid models of gastrointestina cancers in basic and translational research. Nat Rev Gastroenterol Hepatol. 2020;17(4):203-22.

3. Bray F, Ferlay J, Soerjomataram I, Siegel RL, Torre LA, Jemal A. Global cancer statistics 2018: GLOBOCAN estimates of incidence and mortality worldwide for 36 cancers in 185 countries. CA Cancer J Clin. 2018;68(6):394-424.

4. Lutz MP, Zalcberg JR, Ducreux M, Adenis A, Allum W, Aust D, Carneiro F, Grabsch HI, Laurent-Puig P, Lordick F, et al. The 4th St. Gallen EORTC Gastrointestinal Cancer Conference: controversial issues in the multimodal primary treatment of gastric, junctional and oesophageal adenocarcinoma. Eur J Cancer. 2019;112:1-8.

5. Chen W, Zheng R, Baade PD, Zhang S, Zeng H, Bray F, Jemal A, Yu XQ, He J. Cancer statistics in China, 2015. CA Cancer J Clin. 2016;66(2):115-32.

6. Song Y, Ye M, Zhou J, Wang Z, Zhu X. Targeting E-cadherin expression with small molecules for digestive cancer treatment. Am J Transl Res. 2019;11(7):3932-44

7. Citron F, Segatto I, Vinciguerra GLR, Musco L, Russo F, Mungo G, D'Andrea S, Mattevi MC, Perin T, Schiappacassi M, et al. Downregulation of miR-223 expression is an early event during mammary transformation and confers resistance to CDK4/6 inhibitors in luminal breast cancer. Cancer Res. 2020;80(5):1064-77.

8. Liu Y, Feng J, Sun M, Yang G, Yuan H, Wang Y, Bu Y, Zhao M, Zhang S, Zhang X. Long non-coding RNA HULC activates HBV by modulating HBx/STAT3/miR-539/APOBEC3B signaling in HBV-related hepatocellular carcinoma. Cancer Lett. 2019:454:158-70.

9. Mukohyama J, Isobe T, Hu Q, Hayashi T, Watanabe T, Maeda M, Yanagi H, Qian X, Yamashita K, Minami H, et al. miR-221 targets QKI to enhance the tumorigenic capacity of human colorectal cancer stem cells. Cancer Res. 2019;79(20):5151-8.

10. Tsai MM, Wang CS, Tsai CY, Huang HW, Chi HC, Lin YH, Lu PH, Lin KH. Potential diagnostic, prognostic and therapeutic targets of MicroRNAs in human gastric cancer. Int J Mol Sci. 2016;17(6):945.

11. Jiang K, Xie LF, Xiao TZ, Qiu MY, Wang WL. MiR-181d inhibits cell proliferation and metastasis through PI3K/AKT pathway in gastric cancer. Eur Rev Med Pharmacol Sci. 2019;23(20):8861-9.

12. Xu XL, Guo AX, Pan QY, Chang AL, Zhao CR. MiR-99a suppresses cell migration and invasion by regulating IGF1R in gastric cancer. Eur Rev Med Pharmacol Sci. 2019;23(17):7375-82.

13. Shang JC, Yu GZ, Ji ZW, Wang XQ, Xia L. MiR-105 inhibits gastric cancer cells metastasis, epithelial-mesenchymal transition by targeting SOX9. Eur Rev Med Pharmacol Sci. 2019;23(14):6160-9.

14. Wang M, Qiu R, Gong Z, Zhao X, Wang T, Zhou L, Lu W, Shen B, Zhu W, Xu $W$. miR-188-5p emerges as an oncomiRNA to promote gastric cancer cell proliferation and migration via upregulation of SALL4. J Cell Biochem. 2019;120(9):15027-37.

15. Zhou QY, Peng PL, Xu YH. MiR-221 affects proliferation and apoptosis of gastric cancer cells through targeting SOCS3. Eur Rev Med Pharmacol Sci. 2019:23(21):9427-35.

16. Du W, Tang H, Lei Z, Zhu J, Zeng Y, Liu Z, Huang JA. miR-335-5p inhibits TGF-beta1-induced epithelial-mesenchymal transition in non-small cell lung cancer via ROCK1. Respir Res. 2019;20(1):225.

17. Jia Q, Ye L, Xu S, Xiao H, Xu S, Shi Z, Li J, Chen Z. Circular RNA 0007255 regulates the progression of breast cancer through miR-335-5p/SIX2 axis. Thorac Cancer. 2020;11(3):619-30.

18. Liang H, Zhang C, Guan H, Liu J, Cui Y. LncRNA DANCR promotes cervical cancer progression by upregulating ROCK1 via sponging miR-335-5p. J Cell Physiol. 2019;234(5):7266-78

19. Zare A, Ahadi A, Larki P, Omrani MD, Zali MR, Alamdari NM, Ghaedi H. The clinical significance of miR-335, miR-124, miR-218 and miR-484 downregulation in gastric cancer. Mol Biol Rep. 2018;45(6):1587-95.

20. Yuan $\mathrm{HL}$, Wang T, Zhang KH. MicroRNAs as potential biomarkers for diagnosis, therapy and prognosis of gastric cancer. Onco Targets Ther. 2018;11:3891-900.

21. Bartel DP. MicroRNAs: target recognition and regulatory functions. Cell. 2009;136(2):215-33.

22. Liu B, Li J, Cairns MJ. Identifying miRNAs, targets and functions. Brief Bioinform. 2014;15(1):1-19.

23. Kahraman M, Roske A, Laufer T, Fehlmann T, Backes C, Kern F, Kohlhaas J, Schrors H, Saiz A, Zabler C, et al. MicroRNA in diagnosis and therapy monitoring of early-stage triple-negative breast cancer. Sci Rep. 2018;8(1):11584.

24. Kunde SA, Rademacher N, Tzschach A, Wiedersberg E, Ullmann R, Kalscheuer VM, Shoichet SA. Characterisation of de novo MAPK10/JNK3 truncation mutations associated with cognitive disorders in two unrelated patients. Hum Genet. 2013;132(4):461-71.

25. Yoo KH, Park YK, Kim HS, Jung WW, Chang SG. Identification of MAPK10 as a novel epigenetic marker for chromophobe kidney cancer. Pathol Int. 2011;61(1):52-4.

26. Zhang L, Li H, Yuan M, Li M, Zhang S. Cervical cancer cells-secreted exosomal microRNA-221-3p promotes invasion, migration and angiogenesis of microvascular endothelial cells in cervical cancer by down-regulating MAPK10 expression. Cancer Manag Res. 2019;11:10307-19.

27. Dai F, Zhang Y, Chen Y. Involvement of miR-29b signaling in the sensitivity to chemotherapy in patients with ovarian carcinoma. Hum Pathol. 2014:45(6):1285-93.

28. Emami SS, Nekouian R, Akbari A, Faraji A, Abbasi V, Agah S. Evaluation of circulating miR-21 and miR-222 as diagnostic biomarkers for gastric cancer. J Cancer Res Ther. 2019;15(1):115-9.

29. Xia L, Zhang D, Du R, Pan Y, Zhao L, Sun S, Hong L, Liu J, Fan D. miR-15b and miR-16 modulate multidrug resistance by targeting BCL2 in human gastric cancer cells. Int J Cancer. 2008;123(2):372-9.

30. Feng R, Chen X, Yu Y, Su L, Yu B, Li J, Cai Q, Yan M, Liu B, Zhu Z. miR-126 functions as a tumour suppressor in human gastric cancer. Cancer Lett. 2010;298(1):50-63.

31. Kogo R, Mimori K, Tanaka F, Komune S, Mori M. Clinical significance of miR-146a in gastric cancer cases. Clin Cancer Res. 2011:17(13):4277-84.

32. Slattery ML, Herrick JS, Mullany LE, Valeri N, Stevens J, Caan BJ, Samowitz W. Wolff RK. An evaluation and replication of miRNAs with disease stage and colorectal cancer-specific mortality. Int J Cancer. 2015;137(2):428-38.

33. Wang $K$, Jin W, Song Y, Fei X. LnCRNA RP1 1-436H11.5, functioning as a competitive endogenous RNA, upregulates $B C L-W$ expression by sponging miR-335-5p and promotes proliferation and invasion in renal cell carcinoma. Mol Cancer. 2017:16(1):166.

34. Tang H, Zhu J, Du W, Liu S, Zeng Y, Ding Z, Zhang Y, Wang X, Liu Z, Huang J. CPNE1 is a target of miR-335-5p and plays an important role in the pathogenesis of non-small cell lung cancer. J Exp Clin Cancer Res. 2018;37(1):131.

35. Wang Y, Zeng X, Wang N, Zhao W, Zhang X, Teng S, Zhang Y, Lu Z. Long noncoding RNA DANCR, working as a competitive endogenous RNA promotes ROCK1-mediated proliferation and metastasis via decoying of miR-335-5p and miR-1972 in osteosarcoma. Mol Cancer. 2018;17(1):89.

36. Luo L, Xia L, Zha B, Zuo C, Deng D, Chen M, Hu L, He Y, Dai F, Wu J, et al. miR-335-5p targeting ICAM-1 inhibits invasion and metastasis of thyroid cancer cells. Biomed Pharmacother. 2018:106:983-90. 
37. Zhang D, Yang N. MiR-335-5p inhibits cell proliferation, migration and invasion in colorectal cancer through downregulating LDHB. J BUON. 2019;24(3):1128-36.

38. Spring LM, Wander SA, Andre F, Moy B, Turner NC, Bardia A. Cyclindependent kinase 4 and 6 inhibitors for hormone receptor-positive breast cancer: past, present, and future. Lancet. 2020;395(10226):817-27.

39. Oner M, Lin E, Chen MC, Hsu FN, ShazzadHossainPrince GM, Chiu KY, Teng CJ, Yang TY, Wang HY, Yue CH, et al. Future aspects of CDK5 in prostate cancer: from pathogenesis to therapeutic implications. Int J Mol Sci. 2019;20(16):3881.

40. Jia W, Zhao X, Zhao L, Yan H, Li J, Yang H, Huang G, Liu J. Non-canonical roles of PFKFB3 in regulation of cell cycle through binding to CDK4. Oncogene. 2018;37(13):1685-98.

41. Abbosh C, Swanton C, Birkbak NJ. Circulating tumour DNA analyses reveal novel resistance mechanisms to CDK inhibition in metastatic breast cancer. Ann Oncol. 2018;29(3):535-7.

42. Bandi N, Vassella E. miR-34a and miR-15a/16 are co-regulated in nonsmall cell lung cancer and control cell cycle progression in a synergistic and Rb-dependent manner. Mol Cancer. 2011;10:55.

43. Liu Q, Fu H, Sun F, Zhang H, Tie Y, Zhu J, Xing R, Sun Z, Zheng X. miR-16 family induces cell cycle arrest by regulating multiple cell cycle genes. Nucleic Acids Res. 2008;36(16):5391-404
44. Zhao H, Zhang D, Yang L, Wang E. p0071 interacts with E-cadherin in the cytoplasm so as to promote the invasion and metastasis of non-small cell lung cancer. Mol Carcinog. 2018;57(1):89-96.

45. Wu C, Luo K, Zhao F, Yin P, Song Y, Deng M, Huang J, Chen Y, Li L, Lee S, et al. USP20 positively regulates tumorigenesis and chemoresistance through beta-catenin stabilization. Cell Death Differ. 2018;25(10):1855-69.

46. Tournier C, Hess P, Yang DD, Xu J, Turner TK, Nimnual A, Bar-Sagi D, Jones SN, Flavell RA, Davis RJ. Requirement of JNK for stress-induced activation of the cytochrome c-mediated death pathway. Science. 2000;288(5467):870-4.

47. Qiao B, Wang Q, Zhao Y, Wu J. miR-205-3p functions as a tumor suppressor in ovarian carcinoma. Reprod Sci. 2020;27(1):380-8.

48. Li L, Luo Z. Dysregulated miR-27a-3p promotes nasopharyngeal carcinoma cell proliferation and migration by targeting Mapk10. Oncol Rep. 2017;37(5):2679-87.

\section{Publisher's Note}

Springer Nature remains neutral with regard to jurisdictional claims in published maps and institutional affiliations.
Ready to submit your research? Choose BMC and benefit from:

- fast, convenient online submission

- thorough peer review by experienced researchers in your field

- rapid publication on acceptance

- support for research data, including large and complex data types

- gold Open Access which fosters wider collaboration and increased citations

- maximum visibility for your research: over $100 \mathrm{M}$ website views per year

At BMC, research is always in progress.

Learn more biomedcentral.com/submissions 